\title{
Urbanisation et urbanité au Sahara
}

\section{Saïd Belguidoum}

\section{Résumé}

Comprendre l'urbanisation au Sahara, aujourd'hui, implique une mise en perspective historique du phénomène, car celui-ci est tout à la fois en continuité avec les réseaux urbains de l'époque caravanière, et en même temps un fait radicalement nouveau, provoqué par les États-Nations et les politiques d'intégration saharienne. Nouvelles activités et nouvelles catégories sociales se sont combinées pour assurer une large recomposition du paysage urbain. Comme ailleurs, la ville usage, celle de l'œuvre et du signe, est devenue la ville échange, celle des promoteurs, de la fonctionnalité et de la segmentation. L'urbanisme saharien en sort métamorphosé.

\begin{abstract}
To understand urbanization in the Sahara today requires a historical perspective. It represents a continuity of the urban networks of the caravan period, but, at the same time, has elicited a radically new departure, the result of nation-states and their policies of Saharan integration. New activities and social categories have been combined to assure a major reconfiguration of the urban landscape. As elsewhere, the city has become a center of exchange, promotion, functionality and segmentation ; together those elements have brought a metamorphosis to these old Saharan cities.
\end{abstract}

Citer ce document / Cite this document :

Belguidoum Saïd. Urbanisation et urbanité au Sahara. In: Méditerranée, tome 99, 3-4-2002. Le sahara, cette «autre Méditerranée» (Fernand Braudel) pp. 53-64;

doi : 10.3406/medit.2002.3261

http://www.persee.fr/doc/medit_0025-8296_2002_num_99_3_3261

Document généré le 13/06/2016 


\title{
III - LES DYNAMIQUES URBAINES
}

\section{Urbanisation et urbanité au Sahara}

\author{
Urbanization and urbanity in the Sahara
}

\begin{abstract}
Résumé - Comprendre l'urbanisation au Sahara, aujourd'hui, implique une mise en perspective historique du phénomène, car celui-ci est tout à la fois en continuité avec les réseaux urbains de l'époque caravanière, et en même temps un fait radicalement nouveau, provoqué par les États-Nations et les politiques d'intégration saharienne. Nouvelles activités et nouvelles catégories sociales se sont combinées pour assurer une large recomposition du paysage urbain. Comme ailleurs, la ville usage, celle de l'œuvre et du signe, est devenue la ville échange, celle des promoteurs, de la fonctionnalité et de la segmentation. L'urbanisme saharien en sort métamorphosé.
\end{abstract}

L'urbanisation au Sahara est un phénomène problématique à plus d'un titre. Son originalité historique et son devenir actuel obligent à un regard synthétique mettant en relief les dynamiques qui le caractérisent. Notre analyse portera sur le Sahara algérien, pays ou les processus de construction de l'État-Nation ont eu des effets profonds.

L'Algérie vit depuis une quarantaine d'années un mouvement d'urbanisation massif. Pays essentiellement rural avant son accession à l'indépendance, il est engagé dans un processus d'urbanisation, certes inachevé, mais rapide et générateur de nouvelles configurations qui en font un des faits dominants des mutations sociétales actuelles. Cette croissance urbaine permanente $(25 \%$ de la population en $1954,59 \%$ en 1998 ) touche avec la même ampleur les régions sahariennes mais avec des tendances différentes. Les taux de croissance et d'urbanisation très soutenus dépassent la moyenne nationale, et le croît démographique est plus fort que le reste du pays (de 1987 à 1998 la population du Sahara passe de $8,7 \%$ à près de $10 \%$ de la population algérienne).

La structure du réseau urbain saharien est caractérisée par une forte concentration dans les

\begin{abstract}
To understand urbanization in the Sahara today requires a historical perspective. It represents a continuity of the urban networks of the caravan period, but, at the same time, has elicited a radically new departure, the result of nation-states and their policies of Saharan integration. New activities and social categories have been combined to assure a major reconfiguration of the urban landscape. As elsewhere, the city has become a center of exchange, promotion, functionality and segmentation; together those elements have brought a metamorphosis to these old Saharan cities.
\end{abstract}

agglomérations principales au détriment des centres petits et moyens (tabl. 1). Hormis Biskra où le réseau urbain présente des similitudes avec les modèles du Nord, cette distorsion s'explique en partie par les contingences écologiques, mais interpelle sur ses causes, sa dynamique et ses effets.

Qu'est-ce qui fonde un tel phénomène, quelles sont les activités qui sont à l'origine de la concentration urbaine? Les transformations de l'espace renvoient-elles à une urbanisation saharienne ou une urbanisation au Sahara' ${ }^{\prime}$ ' S'agit-il de facteurs endogènes (les villes existantes produisant ellesmêmes les conditions de leur croissance) ou de facteurs exogènes liés aux politiques de développement initiées par le pouvoir central ? Quelles en sont les conséquences sur les modes de vie, quelles formes d'urbanité cela engendre-t-il ?

Pour répondre à ces questions nous présenterons la cité saharienne à travers l'histoire, ce qui en fait sa singularité et ses fondements. Puis nous nous attellerons à identifier les facteurs qui ont concouru aux transformations actuelles. Enfin nous esquisserons un questionnement sur les effets que produit le processus actuel d'urbanisation sur les modes de vie, l'urbanité et les façons de vivre la ville ${ }^{2}$.

\footnotetext{
* Sociologue, Université de la Méditerranée, Département gestion urbaine, Aix-en-Provence.
} 
TABL. 1 - URBANISATION DU SAHARA

\begin{tabular}{|c|c|c|c|c|c|c|c|}
\hline Wilaya & $\begin{array}{l}\text { Population } \\
\text { urbaine }\end{array}$ & $\begin{array}{c}\text { Taux } \\
\text { d'urbanisation }\end{array}$ & $\begin{array}{c}\text { Croissance } \\
\text { urbaine en \% } \\
(1987-1998)\end{array}$ & $\begin{array}{c}\text { Nombre } \\
\text { d'agglomération }\end{array}$ & +100000 & $\begin{array}{l}+50000 a ̀ \\
-100000\end{array}$ & $\begin{array}{l}+5000 \text { à } \\
-50000\end{array}$ \\
\hline Ouargla & 334600 & 75,1 & 3,8 & 53 & 2 & 0 & 13 \\
\hline Biskra & 333200 & 57,9 & 2,9 & 71 & 1 & 0 & 17 \\
\hline El Oued & 325600 & 62,6 & 3,7 & 84 & 1 & 0 & 21 \\
\hline Ghardaïa & 276400 & 92,0 & 4,2 & 22 & 1 & 0 & 9 \\
\hline Béchar & 172400 & 76,5 & 2,0 & 41 & 1 & 0 & 4 \\
\hline Tamanrasset & 92300 & 67,3 & 15,7 & 27 & 0 & 1 & 2 \\
\hline Adrar & 75100 & 24,1 & 3,6 & 149 & 0 & 0 & 8 \\
\hline Tindouf & 25000 & 92,3 & 14,9 & 2 & 0 & 0 & 1 \\
\hline Illizi & 12600 & 36,9 & 5,9 & 16 & 0 & 0 & 2 \\
\hline Total & 1597000 & 68,0 & & 465 & 6 & 1 & 76 \\
\hline
\end{tabular}

Source : Armature urbaine, Collections statistiques, ${ }^{\circ}$ 97, ONS 1998, Alger $^{3}$

Les processus agissant aujourd'hui sur l'ensemble de la société sont une nouvelle illustration des vicissitudes d'une longue histoire urbaine jalonnée par des périodes de grandeur et de déclin, de discontinuités et de ruptures. Pourtant à travers cette histoire agitée, les cités du Sud font figure d'exception en traversant les siècles avec une remarquable pérennité.

\section{1 - LES CITÉS SAHARIENNES À TRAVERS L'HISTOIRE : UNE MISE EN PERSPECTIVE}

\subsection{Permanence spatiale et structure du réseau oasien}

C'est avec l'essor du commerce caravanier que les cités tout en se développant ont attiré l'attention des voyageurs et géographes. Situées sur les grands axes de communication, notamment N-S et $\mathrm{W}-\mathrm{E}$, leur importance est régulièrement évoquée

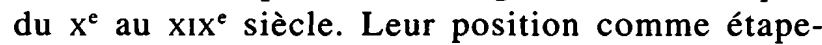
relais leur confère une fonction forte, enjeu constant de contrôle durant dix siècles. Les différents conflits qui ont jalonné l'histoire du Sahara, à caractère religieux ou ethnique, ont souvent eu comme cause réelle le contrôle des axes caravaniers. Mais leur raison d'être ne se limite pas à la seule fonction de centres-relais; elles regroupent des populations fixes, sont en relation avec les tribus nomades, et vivent de l'exploitation des palmeraies. Leurs fortes caractéristiques spatiales, économiques et sociales font d'elles des agglomérations originales.

La première caractéristique repose sur leur organisation spatiale et les modes d'appropriation territoriale. C'est avec les mêmes termes que, d'El BeKri (1913) au Docteur Shaw (1980), elles seront décrites durant dix siècles. Ce sont des chapelets d'agglomérations, villages, bourgs et bourgades s'égrenant le long des oueds. Parmi cet ensemble d'agglomérations, une cité s'impose aux autres par son importance et son rôle.

En fait, au gré des événements politiques ou des contingences physiques, notamment hydrauliques, les villages se forment et peuvent disparaître. Les luttes entre cités, les expéditions des pouvoirs centraux ont comme conséquence l'émergence de nouveaux villages ou au contraire leur destruction. L'existence de ruines et de restes de palmeraies abandonnées, constatées aux différentes époques et dont les traditions locales conservent le nom des ksour aujourd'hui disparus, atteste de ces mouvements. Inversement, de nouvelles installations de populations peuvent participer à la dynamique des réseaux. Tel a été le cas pour le Souf qui, longtemps marginalisé et dépourvu de véritables cités, confiné à une fonction de refuge, commencera à partir du $\mathrm{XVII}^{\mathrm{e}}$ siècle à développer sa propre organisation.

Nous ne savons pas grand chose sur la taille de ces agglomération ${ }^{4}$, mais ce sont les rôles qu'elles jouent qui les qualifient. Continuellement tiraillées entre «la vie bédouine et la vie citadine» (Berque, 1958), les cités ont néanmoins des traits forts qui les différencient de leurs ksour. La configuration générale de tout regroupement stable au Sahara prend la forme du ksar, cet ensemble de bâtis de taille variable autour duquel se développe une palmeraie. Mais les statuts de ces agglomérations les distinguent qualitativement, et une hiérarchie apparaît entre le «ksar cité» et le «ksar village».

Le village est une agglomération durable, de taille relativement modeste, qui existe par et pour son terroir. L'aire villageoise est une totalité, qui souvent se laisse lire à travers les correspondances qui existent entre la configuration des quartiers bâtis et celle des quartiers de culture. Cette myriade de petites agglomérations, qui jalonnent les différentes vallées, revêt une dimension villageoise. Quand un ksar plus important émerge, il s'érige comme cité et devient le pôle structurant d'un réseau. Entre le ksar village et le ksar cité des ruptures et des passages existent.

À travers les diverses descriptions historiques, il ressort que les cités et les ksour qui leur sont rattachés ont des tissus structurés à partir de quelques grands équipements et éléments récurrents : la mosquée, les places, les rues, le fort (bordj), les remparts, les marchés, les commerces et l'artisanat. 
La rue est l'artère essentielle de la vie sociale. Avec la place, elle constitue l'espace public, le lieu où l'on passe, où l'on se croise et se rencontre. Les rues sont hiérarchisées et pénètrent la cité en un système complexe de courbes, d'axes principaux, de ruelles, de venelles et d'impasses. Certaines ruelles ou impasses ont un usage semi-public ou semi-privé. D'autres, les rucs marchandes, sont bordées d'échoppes des boutiquiers et artisans. C'est l'importance de ces équipements qui permet de distinguer la cité du village, la médina du ksar. La grande mosquée et l'existence au sein de la même cité d'autres mosquées, l'existence de remparts et de fossés, de portes, de marchés et de commerces sont les éléments de différenciations.

Ce tissu urbain évolue en fonction de la prospérité de la cité. C'est à la fin du $\mathrm{X}_{111^{\mathrm{e}}}$ siècle que Biskra est dotée d'une citadelle. Touggourt est également entourée d'un mur de moellons et d'argile, alors que Ouargla possède un mur d'enceinte en briques crues. Les constructions sont en briques crues séchées au soleil ou en mauvais pisé. Parfois elles sont en pierres, matériau noble réservé aux édifices publics (mosquée et bordj) et aux demeures les plus riches.

Quant à l'urbanisme et à l'architecture, des différences apparaissent. Dans le M'Zab ou à Touggourt, les premiers constructeurs élevèrent leurs demeures autour de la mosquée principale comme centre, les rues dessinant des cercles concentriques. Dans d'autres cités, la création de nouveaux quartiers se fait par rajout, sans respect du schéma initial. La ville se développe selon un modèle qui peut être radio-concentrique (Touggourt, Ngoussa, les cités du M'Zab) ou de forme plus étirée (les cités du Souf). Elle traduit aussi les inégalités sociales, qui s'inscrivent dans le tissu, et maintient sur ses marges les campements temporaires des nomades.

Les fonctions de ces cités se regroupent en trois grands types : le politique, le religieux et l'économique. C'est à partir de ce triptyque qu'il faut comprendre leur permanence, leur raison d'être, leurs moments de gloire et leurs périodes de régression.

\subsection{L'économie des cités : la production agricole et le trafic caravanier}

La première richesse des cités provient de la production dattière dont la qualité et la notoriété en fait une denrée recherchée et permet d'entreprendre un commerce avec l'extérieur, le Nord comme le Sud (El BeKri, 1913). Il permet de subvenir aux besoins élémentaires, mais aussi de s'enrichir. Le commerce et les échanges interrégionaux, parfois lointains, font que ces places du Sahara se diversifient de plus en plus (céréales, tissus, sel, or, cheptel et esclaves) et les mettent en relation avec les villes du Nord et du Sud. Parmi les nombreuses marchandises qui transitent ainsi, le commerce des esclaves est très lucratif. Ce trafic perdurera longtemps, comme le constate T. SHAw au XVIII ${ }^{e}$ siècle.
Le trafic caravanier, c'est aussi le passage des pèlerins qui venant de l'ouest du Maghreb, font étape dans la région. À la fin du $\mathrm{Xv}^{\mathrm{e}}$ siècle, Touggourt détourne à son profit les caravanes de pèlerins venues de l'ouest (Sijilmasa, Fès, Tlemcen), et ce, au détriment de Ouargla. Ce qui dynamise son économie ; chaque caravane de pèlerins, qui séjourne plusieurs jours, apporte troupeaux, grains et autres marchandises.

Dès le $\mathrm{IX}^{\mathrm{e}}$ siècle, grâce au commerce saharien, Biskra est un centre important. Indépendante de l'empire rostémide, elle supplante Tobna et devient le centre actif d'une province et la concurrente directe de Sedrata (Lethielleux, 1983). Au Xive siècle Biskra atteint son apogée politique mais aussi économique, grâce au commerce caravanier. On y reçoit les ambassadeurs du Mali pour traiter des affaires, et ce malgré la concurrence sévère de Ouargla et Touggourt.

Cette intense activité économique, qui articule production agricole et commerce, est contrôlée de très près par une administration politique qui accapare une part importante des richesses, directement (possession de vastes palmeraies, financement du commerce caravanier) ou par le biais de l'impôt. Ces cités ne sont pas de simples villes-relais, elles sont aussi en mesure d'assurer leur propre trafic caravanier.

\subsection{Le champ politique : un rapport de forces à trois composantes}

Le champ politique (Bourdieu, 1980) est constitué et structuré autour de trois grands types d'agents : le pouvoir central (représenté par les différentes dynasties, puis la régence d'Alger, puis la colonisation française), le pouvoir local exercé dans les cités (l'aristocratie locale, les théologiens et la bourgeoisie foncière), les tribus bédouines qui occupent les territoires attenants aux palmeraies. Ce champ politique est régulièrement agité par des luttes incessantes où tour à tour s'allient ou s'affrontent ces composantes, à travers un jeu complexe de rapports (cités entre elles, cités et pouvoir central, cités et tribus bédouines).

L'histoire politique de ces cités, ce sont d'abord les rapports qui s'établissent avec le pouvoir central. Rapport de domination, exprimé par les allégeances et le versement d'un tribut annuel; rapport d'alliance, lorsque le pouvoir central, menacé par d'autres dynasties, a besoin du renfort économique et militaire des cités ; rapport conflictuel, lorsque les cités cherchent à s'affranchir de la tutelle du pouvoir central. Ces relations multiples sont brouillées par les rapports de forces locaux, eux-mêmes structurés autour des appartenances religieuses et claniques, les rivalités entre les différents réseaux de ksour ou l'opposition entre la cité et les pasteurs nomades. 
Les cités et leur territoire d'influence sont administrées par de puissantes familles, celles de l'aristocratie terrienne et marchande, qui tirent leur légitimité de la position qu'elles occupent dans les autres champs de la vie sociale : le champ religieux (conférant un puissant capital symbolique) ; l'économique (contrôle de la production et du commerce), le politique (position vis-à vis du pouvoir central et des tribus bédouines) et l'importance de leur capital social (populations sur lesquelles elles s'appuient et qui les soutiennent). Avant que ces contrées ne s'érigent en petit royaume (période des sultanats locaux), une cité comme Biskra est gérée par un conseil administratif au sein duquel siègent les représentants des principales familles. Le pouvoir d'une famille se transmet d'une génération à l'autre, mais il peut se produire des renversements. L'ascension au XIV ${ }^{\mathrm{e}}$ siècle des Beni Mozni à Biskra et des Ben Djellab à Touggourt, illustre comment un clan construit sa domination : la légitimité (origine prétendue hilaliennne ou chérifienne), la richesse (possession de grandes palmeraies, contrôle caravanier), les alliances (IBN Khaldoun, 1982).

Durant des siècles ces régions sont l'enjeu de luttes politiques intenses opposant les grandes familles qui s'appuient tour à tour sur les dynasties du Maghreb et les tribus nomades du Sahara pour imposer leur hégémonie. Mais ces alliances sont souvent tactiques et précaires. Les rapports de force fluctuent entre la trilogie constituée par le pouvoir central, l'aristocratie des cités, et les tribus bédouines. Chaque cité essaie d'établir son indépendance et de contrôler sa région et les routes caravanières.

Ces rapports entre la ville et l'agriculture, la cité et les villages, les citadins et les bédouins sont inégaux. La cité a besoin de l'agriculture et de l'activité des bédouins pour exister et capte une partie de leurs richesses. D'où les incessantes révoltes des tribus nomades contre la ville oppressante et opulente, d'où aussi les divorces liés au fait que la ville est capable de tirer ses richesses en investissant loin de ses remparts, en finançant par exemple, les commerces lointains.

Avec la période turque, le Sahara connaît de nouvelles turbulences. Dans l'Est, le Beylick de Constantine s'appuie sur Biskra pour s'assurer le contrôle de la région dont les populations ne reconnaîtront que par la force cette nouvelle souveraineté..$^{5}$ Tout le XVII ${ }^{\mathrm{e}}$ siècle est marqué par les luttes entre les cités d'une part et les cités et les tribus bédouines d'autre part. L'économie de la région, la prospérité des cités et des bourgades en souffrent énormément. Les axes commerciaux sont détournés vers le M'Zab ou vers l'Égypte.

\section{la vie sociale \\ 1.4. Le champ religieux : la légitimation de}

Le religieux légitime l'ensemble des autres champs. Le droit canonique surveille les activités des princes, des marchands et des producteurs. La vie religieuse a des impacts profonds, tant sur le pouvoir politique, sur la distribution sociospatiale entre les ksour et au sein des cités, que sur la vie économique.

À la fonction proprement théologique, celle de l'étude, de la production du droit canonique nouveau, d'écrits religieux importants, s'ajoute celle du contrôle social qui s'affirme et marque la vie des cités. Le contrôle religieux s'applique sur la morale citadine comme sur les transactions commerciales ${ }^{6}$ en définissant le licite et l'illicite, le pur et l'impur. Tout en surveillant et contrôlant les deux autres ordres, il vit de leurs dons. Ce qui lui permet d'exercer son pouvoir sur les biens immobiliers et le foncier, les biens habous.

L'histoire des cités sahariennes nous montre qu'à la mosaïque des peuplements correspondait une diversité d'obédiences religieuses et de communautés qui cohabitaient et parfois entraient en lutte (Ibadites et Sunnites, ordres confrériques). Avec le développement du confrérisme, elles accueillent où produisent elles-mêmes des zaoü̈as ${ }^{7}$ dont certains cheikhs ont une grande influence sur le plan local (chaque oasis est protégée par un saint) et parfois à l'échelle du Maghreb et de l'Afrique sahélienne. Temacine, grand centre de la Tidjanya au XIX ${ }^{e}$ siècle, en est un exemple.

\section{sociale \\ 1.5. La vie dans la cité et la structuration}

Occupée originellement par des populations berbères, le Sahara accueille par vagues successives des peuplements différents. Ces apports nouveaux, permanents ou provisoires, participent pleinement à la vie économique, sociale, politique et religieuse de ces contrées.

Quand El HaUQAL visite la région, il identifie ses habitants comme des Berbères. L'influence de la période romaine semble encore vivace jusqu'au XII $^{\mathrm{e}}$ siècle (EL BEKRI, 1913). Puis divers peuplements arabes venus au $x^{e}$ siècle avec les Hilaliens, les Hariz, les Maghraoua (Beni Sindja et Righa) les Chaamba, les Dawawida (XIII ${ }^{\mathrm{e}}$ siècle), les Troud $\left(X^{\prime} v^{e}\right.$ siècle), les populations harratin (métayers des palmeraies venus d'Afrique noire) et bien d'autres donneront une nouvelle configuration au Sahara, véritable mosaïque de peuplement. Les migrations des différents groupes zénètes et sahandja, leur sédentarisation, leur fusion ou intégration dans des groupements existants participent à la construction de collectivités historiques, s'identifiant à des généalogies qui prennent corps à travers des horma, que les légendes locales légitiment. Entre le Xvi et le $\mathrm{XVIII}^{\mathrm{e}}$ siècle de nouvelles populations s'installent et se fondent dans les groupes déjà existants. Origines réelles ou mythiques, ces appartenances revendiquées détermineront longtemps les lignages, les alliances et les relations sociales. 
À cette diversité du peuplement, correspond une distribution spatiale particulière qui se retrouve non seulement entre les réseaux de ksour, mais à l'intérieur même des réseaux et des cités. Souvent le tissu des cités reproduit et traduit les discriminations familiales et religieuses et l'existence de minorités, bénéficiant de statuts particuliers dans la cité. Sunnites dans les citćs du M'Zab, Ibadites dans celles de l'oued Righ, Harratin et Hachachna, en sont des exemples. Pendant des siècles, des minorités juives se sont maintenues dans le Sahara (Laghouat, Bou Saada, M'Zab, Touat, Zab) ${ }^{8}$ et ont marqué la vie sociale des cités, notamment en se spécialisant dans l'artisanat et le commerce.

La distinction entre le monde du travail et celui de l'élite sociale, politique et culturelle est organisée à partir des trois grands ordres : l'aristocratie ou la bourgeoisie foncière et marchande, les religieux (imams, cadis et docteurs de la foi), et la masse du petit peuple. Ces derniers sont constitués des petits artisans et commerçants, petits propriétaires et exploitants, ouvriers et métayers des palmeraies attenantes aux cités, journaliers et tous ceux qui s'occupent des fonctions afférentes à la vie de la cité (fonctions publiques ou fonctions privées). C'est-à-dire les métiers liés à l'entretien de la cité, la perception de l'impôt, les portefaix nécessaires à l'activité marchande, et bien d'autres encore dans des cités qui devaient surveiller leur arrière-pays, se défendre contre les intrusions des tribus nomades ou contre les cités voisines, organiser la protection des caravanes ainsi que les financer, stocker les marchandises, arbitrer les différends (voisinage, transaction), faire respecter l'ordre, organiser la distribution de l'eau et régler les conflits que cela pouvait engendrer.

C'est le faible niveau de vie qui oblige les gens du Sud à émigrer vers le Nord et notamment à Alger. Aux $\mathrm{XVII}^{\mathrm{e}}$ et $\mathrm{XVIII}^{\mathrm{e}}$ siècles, la présence des «minorités venues $d u$ Sud» est signalée dans différents corps de métiers (portefaix, gardiens de nuit, revendeurs d'eau, colporteurs), dans le cadre souvent d'une émigration de courte durée. On retrouve des Soufis à Tunis et dans les palmeraies du Zab et de Tozeur, comme saisonniers (BEN Mansour, 1998 ; Venture de Paradis, 1983).

\subsection{Une urbanité de signes}

La qualification urbaine des cités ne repose pas sur leurs seuls éléments quantitatifs. L'équipement rituel, la mosquée à prône hebdomadaire et les écoles coraniques, les rues marchandes et les places, propices à la communication, font partie des éléments constitutifs de la cité. C'est ce seuil qualitatif qui définit la citadinité. L'urbanité a pour socle un système économique de production et d'échange, un rôle politique et symbolique fort qui se matérialise par un complexe architectural, induisant des modes de vie propres, des inscriptions spatiales de pratiques sociales particulières et spécifiques. Les sociétés sahariennes en s'appropriant leur espace, l'aménagent, le façonnent et le structurent selon leurs besoins. L'espace, support de leurs activités, est aussi le reflet de leur organisation sociale. L'architecture banale et monumentale est l'expression de cette appropriation spatiale.

La cité est un lieu de production de signes que l'architecture, en s'inscrivant durablement dans l'espace, traduit en devenant référence symbolique pour le citadin comme pour le bédouin dont le rapport avec la cité est constant. Le décalage qui oppose de part et d'autre des remparts, le citadin et le rural n'est pas seulement politique et économique, il est aussi social et culturel. Le langage, les habitudes et modes de vie, l'histoire et la mémoire collective, les tenues vestimentaires comme les pratiques culinaires sont autant d'éléments de distinction qui ont participé à la fabrication de la citadinité saharienne. La cité se distingue de la bourgade dans le sens où elle est un urbanisme de signes; signes religieux, politiques ou économiques, que l'architecture réifie en organisant les espaces (public/privé, sacré/profane).

La cité repose sur un ordre multifonctionnel : le politique, le religieux, le droit, l'étude, l'artisanat, le commerce (BERQUe, 1958). Ce qui est remarquable, c'est l'articulation et l'imbrication de ces différentes fonctions. Si le politique commande, le commerçant, l'artisan et le paysan produisent la prospérité, le religieux contrôle et légitime l'ensemble des fonctions. Toute l'éthique citadine se résume dans un symbole social puissant: la mosquée et son minaret d'où résonne l'appel à la prière, qui rythme le cycle quotidien et hebdomadaire, élément central de cet urbanisme de signes qui donne «signification et signalisation» à la cité.

\section{2 - L'ÉTAT-NATION ET LES NOUVELLES FORMES D'INTÉGRATION DE LA VILLE SAHARIENNE À LA SOCIÉTÉ GLOBALE}

\subsection{Transformations globales et nouvelles configurations sahariennes}

Presque immuable dans sa structure, son organisation et ses fonctions durant des siècles, la ville saharienne vit depuis une quarantaine d'années des mutations qui bouleversent en profondeur ses structures. Illustrées par les données économiques, sociales et démographiques ces transformations ne sont pas seulement quantitatives, c'est la nature même de la ville saharienne qui se modifie.

Ces changements ont incontestablement une dimension exogène, liée à une volonté étatique de 
prendre en charge les territoires sahariens pour $y$ diffuser sa logique de développement. Mais la ville saharienne est aussi mue par une dynamique endogène. Certes si les agents et groupes sociaux sont agis avant d'agir, ils sont aussi porteurs de stratégies dont le poids est indéniable dans les mutations en cours. La ville est aujourd'hui le résultat, la rencontre entre ces deux types d'intervention, ces deux types de logique.

La colonisation française avait déjà intégré progressivement le Sud dans une logique globale de société (création des territoires administratifs et militaires du Sud, puis de deux départements). C'est surtout avec la réorganisation des circuits commerciaux que les conséquences se font sentir. $\mathrm{Au} \mathrm{XIX}^{\mathrm{e}}$ siècle, la disparition d'une des fonctions essentielles de l'économie des cités, le commerce transsaharien, confine l'activité aux fonctions agricoles (palmeraies et transhumances traditionnelles). L'appauvrissement des cités oblige les populations à émigrer vers le Nord, souvent dans un cadre saisonnier ou de courte durée, pour aller chercher les revenus que l'économie locale ne fournit plus. Ce mouvement migratoire qui remonte à la période turque s'est accru, revêtant des formes différentes selon les groupes et les cités sahariennes. L'émigration mozabite est une émigration de commerçants. Il en est de même pour les Soufis, qui profitant de facteurs tels que frontières nationales et pénuries liées à la Deuxième Guerre mondiale, mettent en place un réseau commercial à travers le Tell et la Tunisie. Mais pour la grande majorité des migrants, il s'agit d'une émigration de travail et de misère.

Avec la construction de l'État-Nation, engagée depuis l'indépendance, l'intégration à la société globale prend une forme différente. Le Sud revêt une importance stratégique pour le nouveau pouvoir central. Les gisements pétrolifères et leur mise en exploitation (1956), montrent l'ampleur des richesses économiques qu'il recèle. C'est aussi au niveau politique et symbolique que l'importance de ces territoires est perçue : la nation algérienne intègre la dimension saharienne dans son système de représentation. La politique administrative et d'investissement a pour but d'affirmer cette souveraineté sur un Sahara, partie intégrante de la Nation et donc objet des mêmes politiques.

En l'espace de vingt ans, le Sahara se modifie profondément. Sous l'impulsion d'une politique volontariste, en généralisant la prospection pétrolière, en créant des entités administratives nouvelles, en investissant dans l'économie et les équipements, le Sahara change de visage, l'urbain devient un fait dominant, les ressources économiques se multiplient, de nouveaux groupes sociaux émergent.

Le découpage administratif et l'investissement économique sont les instruments clés de la politique d'intégration à la société globale. Le Sahara est découpé en neuf wilayas (contre deux en 1962),
50 daïras et 165 communes (tabl. 1). Cette nouvelle trame de l'organisation territoriale permet la création de nombreuses activités, d'équipements et de services (directions et sous-directions administratives, hôpitaux, centres de santé, lycées, collèges, universités, réseaux de postes et de banques) dont les conséquences sur l'emploi, l'habitat, les modes de vie seront grandes. L'exploitation pétrolière et la création des deux grandes bases (Hassi-Messaoud et Hassi Rmel) génèrent une dynamique nouvelle. Enfin les grands travaux d'infrastructures routières et aéroportuaires facilitent les échanges et la mobilité dans le Sahara et avec le reste du pays.

En faisant du Sahara un territoire d'intégration politique et économique, l'État modifie les donnes de la société saharienne. La diffusion d'un modèle de développement et de nouvelles logiques (forte présence de l'État, salarisation croissante, migrations de travail, création de nouveaux bassins d'emploi) rencontre un dynamisme interne reposant sur les ressources propres de la société saharienne. Le déclin des formes traditionnelles de l'économie saharienne a obligé les populations à s'adapter aux exigences de l'économie globale. L'intégration au marché de l'emploi, industriel et tertiaire, l'adaptation aux nouveaux réseaux commerciaux, le développement d'un secteur de PMI, le maintien de l'activité agricole et l'émergence de nouvelles productions témoignent d'un réel dynamisme interne, qui certes se développe à partir des nouvelles opportunités de l'économie, mais qui s'appuie aussi sur les acquis historiques de la société saharienne.

$\mathrm{Au}$ cours des quarante dernières années la croissance des agglomérations du Sud a été spectaculaire. De 1960 à 1998, la ville de Touggourt multiplie sa population par 6, Ghardaïa par 10 , El Oued par 10, Ouargla par 15. Le phénomène est général et touche l'ensemble des agglomérations.

Cette croissance repose sur des forces d'attraction diverses : sédentarisation des nomades, exode rural, inversion des flux migratoires. Historiquement, les populations nomades ont toujours participé au renouvellement de la population des villes. Mais aujourd'hui, cette sédentarisation est massive et définitive. La fin des activités traditionnelles de transhumance oblige ces populations à se fixer dans les villes, lieux où sont concentrées les activités et donc les nouvelles sources de revenus. Ainsi de 1966 à 1977, la proportion de nomades passe de $40 \%$ à $10 \%$ dans la wilaya de Ouargla (BISSON, 1983a). Les nouvelles activités injectées par la société globale ont aussi pour effet l'arrivée de populations du Nord de l'Algérie, marquant l'inversion d'une tendance migratoire séculaire et participant à la nouvelle configuration urbaine.

Cette croissance est aussi spatiale et aboutit parfois à l'absorption des agglomérations voisines. Le cas d'El Oued est illustratif. Une véritable conurbation, annexant à partir d'El Oued, les localités de 
Kouinine, Robbah et Bayadha, constituant une entité spatiale de 154000 habitants sur $20 \mathrm{~km}$ de continuité spatiale, s'est mise en place.

Loin d'être uniformes, ces transformations permettent d'identifier de grands modèles correspondant à des entités régionales. La typologie de J. Bisson (1983b) montre que si l'urbanisation est un phénomène général et global, les types de villes qui en résultent sont mus par des logiques et des formes d'organisation différentes. Trois grands types se dégagent : un Sahara oriental dominé par les hydrocarbures (pays de Ouargla), un Sahara septentrional fief des commerçants (M'Zab, Souf, Ziban) et un Sahara occidental très rural (Gourara, Touat).

Il faut souligner la manière spécifique par laquelle chaque région, chaque ville est entrée dans ce nouveau processus d'intégration à l'économie globale. Selon les cas c'est une combinaison d'impulsions ou un facteur dominant qui sert de moteur au développement urbain : dimension géopolitique à Ouargla et Tamanrasset, celle de l'économie pétrolière à Ouargla, dans le $\mathrm{M}^{\prime} \mathrm{Z}$ ab et à Touggourt, et celle des nouveaux circuits marchands dans le Souf et le M'Zab. L'urbanisation des anciennes cités, l'émergence de nouvelles villes, la transformation plus ou moins profonde des tissus internes, le type de réseau qui se met en place (ville seule, réseau de villes, conurbation), ont comme cause la rencontre entre la politique volontariste et la dynamique privée, produisant une véritable dynamique de synthèse.

\subsection{Une population active de plus en plus différenciée, et une forte salarisation}

La distribution des activités souligne les mutations de la structure économique. Le taux d'activité de la population des villes du Sud est identique à la moyenne nationale $(28 \%)$, la dépassant dans certains cas. L'activité des femmes est même supérieure à la moyenne nationale $(9,6 \%)$, notamment à Béchar $(14,4 \%)$ et Touggourt $(12,7 \%)$, mais reste encore faible à Ghardaïa $(5,7 \%)$ et à El Oued $(5,1 \%)$.

La diversification des activités est notable. En 1980, l'agriculture saharienne occupait $24 \%$ des actifs contre $50 \%$ pour le reste de l'Algérie, l'industrie $18,7 \%$ contre $10 \%$, le BTP $19 \%$ contre $16 \%$ et les services $38 \%$ contre $23 \%$. Selon les villes, la distribution varie, mais la diversification et la salarisation restent des données constantes.

L'impact des emplois étatiques, liés au tertiaire administratif et de services et au secteur productif et de construction est manifeste. À Biskra, le secteur public (collectivités locales et État) emploie $25 \%$ de la population active, dont $29 \%$ dans l'enseignement, $8 \%$ dans la santé, $29 \%$ dans l'industrie et les travaux publics et $34 \%$ dans les services administratifs (Annuaire statistique, 1996). L'importance de ce secteur d'emploi, à forte proportion de cadres moyens et supérieurs, permet d'injecter une masse monétaire dans les circuits de l'économie régionale, impulsant les autres activités.
Si l'implantation du secteur des hydrocarbures est limitée à deux grandes bases, ayant généré la création d'une seule ville nouvelle (Hassi-Messaoud, 37500 habitants, et 17000 emplois), elle a servi de catalyseur à l'activité industrielle, de services et de sous-traitance de nombreuses localités. La zone industrielle de Touggourt, terminal ferroviaire important, et les PMI qui y sont installées, les bases logistiques de la Sonatrach (Société nationale des hydrocarbures), implantées dans de nombreuses villes, sont des exemples de ces retombées.

\subsection{L'activité marchande: du petit} commerce aux grands réseaux régionaux et nationaux

Avec la fin du commerce caravanier, le Sahara a su réagir en adaptant son dynamisme marchand aux nouvelles réalités de l'économie globale. Trois niveaux caractérisent la fonction marchande des villes : le petit commerce, les places régionales, et l'activité marchande à échelle nationale.

Le commerce de détail et les activités de services aux particuliers sont particulièrement développés dans l'ensemble du tissu urbain. À Ouargla on dénombre un commerce pour 23 habitants, à El Oued 1 pour 14 , à Biskra 1 pour 16 . Cette «hypertrophie du commerce sédentaire» (BIsSON, 1983a) n'est pas seulement le signe d'une multiplication d'activités de survie. Elle est aussi l'expression de la «fonction polarisatrice» de centres dont l'attraction est très large, et de l'importante masse monétaire en circulation.

La fonction traditionnelle de marchés hebdomadaires régionaux s'est largement maintenue. Ouargla, Touggourt ont des marchés hebdomadaires qui rayonnent sur leur région respective et sont animés par des gros commerçants qui sillonnent le Sahara. Ces villes maintiennent aussi des marchés quotidiens (souk Bel Abbes à Ouargla, souk Libya à El Oued). Lieu de rencontre des ruraux et des citadins, des habitants des différentes cités appartenant à l'aire régionale de la ville, le marché reste un moment fort de la vie sociale.

L'activité à échelle macro est un des éléments les plus remarquables de l'adaptation à l'économie globale. Le $M^{\prime} Z a b$, où «la citadinité est le produit d'une longue tradition commerciale... a une position privilégiée qui lui permet de jouer un rôle éminent dans l'organisation de l'espace, car c'est à l'échelle saharienne, une plaque tournante très animée» (BIsSON, 1983b). Le Souf est aussi une des places les plus actives, organisant entre autre le commerce frontalier.

\subsection{Le nouveau rapport ville-campagne et} l'adaptation de l'agriculture

Le Sahara vit une modification de son agriculture. À côté d'une production dattière toujours active (malgré la destruction de palmeraies liée à 
l'urbanisation), une agriculture spéculative (maraîchage) et intensive s'est mise en place (Ziban, Souf).

Même si l'activité agricole n'occupe plus une place prépondérante dans les activités, elle joue toujours un grand rôle tant dans la formation des revenus que dans la fonction identitaire de ces régions. Dans les faits, il y a souvent mise en place d'un système familial de polyactivités où l'agriculture continue de subvenir, avec d'autres activités marchandes ou salariales, aux besoins de nombreuses familles.

L'agriculture du Sud, c'est aussi l'émergence d'une petite et moyenne paysannerie de production marchande, qui s'est spécialisée dans le maraîchage. Cette agriculture non seulement alimente les villes mais aussi des marchés de gros, tel celui d'El Ghrouss, dans la wilaya de Biskra, un des premiers marchés maraîchers d'Algérie. Enfin, même marginale, l'existence de grandes exploitations agricoles capitalistes (domaine Daouïa à El Oued, grandes entreprises de la région de Ouargla) annonce un nouveau type d'agriculture.

De manière générale, l'activité agricole est liée à l'économie et à la sociologie des villes. Elle alimente les marchés urbains et permet l'essor d'un artisanat et d'un commerce spécifique. De plus, la forte présence paysanne dans l'habitat urbain, confère aux villes sahariennes une originalité toute particulière. Par l'habitat, les pratiques sociales qu'ils entretiennent, les formes d'appropriation des espaces publics et privés, les paysans citadins participent pleinement à la quotidienneté urbaine. ${ }^{9}$

\subsection{Anciennes et nouvelles élites}

Les mutations en cours modifient la logique de la structuration sociale des sociétés sahariennes, dont l'organisation reposait sur une articulation entre les rapports économiques, les alliances politiques et les appartenances lignagères et claniques. Aux anciennes élites sociales qui combinaient puissance politique (système d'alliance à caractère lignager), puissances économiques (propriété foncière et contrôle du commerce caravanier) et légitimité religieuse, se rajoutent ou se substituent de nouveaux groupes qui ont tiré profit des nouvelles dynamiques sociétales. Selon les régions, leur histoire particulière et les formes de leur intégration à la société globale, de nouvelles figures locales émergent, les anciens groupes dominants s'adaptent et d'anciennes notabilités sont déchues.

Ainsi au M'Zab, le caractère cloisonné et protégé du système social a permis aux élites existantes de se reproduire, même si les transformations structurelles de la société ont servi à la promotion sociale de nouvelles familles. Dans le Souf, les rivalités entre El Oued et Guemar, alimentées par des solidarités lignagères, aboutissent à un partage du champ économique au sein duquel émergent de nouvelles fortunes à dimension nationale et même internationale. La réactivation récente des confréries religieuses (Tidjanya, Rahmanya et Qadirya), un moment marginalisées par le pouvoir central, participe aux enjeux de contrôle du pouvoir local.

Eviter la déchéance sociale, implique pour les élites locales de composer avec le pouvoir central et de s'adapter aux mutations structurelles. La participation au sein de l'appareil administratif local comme national s'inscrit dans cette stratégie; nombreuses sont les grandes familles ayant des membres qui occupent des fonctions importantes dans l'appareil d'État.

Aujourd'hui, la principale source de richesse économique est liée à l'activité commerciale. El Oued et le $M^{\prime}$ Zab sont les cas les plus probants montrant comment l'activité marchande a réussi à générer des réseaux de commerçants puissants, dont une partie de l'accumulation permet un financement d'activités industrielles. Si les Mozabites se sont appuyés sur une longue tradition de réseaux commerciaux et d'entreprises, le cas du Souf est étonnant. Longtemps marginalisés socialement et géographiquement, les Soufis sont à l'origine d'un puissant dynamisme commercial. Les réseaux commerçants développés tout au long du $\mathrm{xx}^{\mathrm{e}}$ siècle ont permis des accumulations qui font de la région une place marchande puissante et un centre de petites et moyennes industries. À Ouargla, les activités commerciales sont animées par des immigrés du Nord, notamment Kabyles, mais surtout par des commerçants mozabites qui ont pris le contrôle de tout un quartier dans le prolongement de Beni Thour.

Le secteur privé est cependant très hétérogène. À côté d'un petit commerce $(60 \%$ du secteur privé à Biskra), qui souvent s'apparente à une activité complémentaire ou de faibles revenus, se développe un secteur de moyens établissements loin d'être marginal. À Biskra, il représente $8 \%$ du secteur privé (800 établissements dans la ville), auquel il faut ajouter les activités de distribution (20\%) et le transport (410 exploitants de lignes de transports en commun). Ces activités réellement lucratives, mises en œuvre par une bourgeoisie marchande, entrepreneurs de bâtiments et petits industriels, caractérisent la nouvelle élite économique.

\subsection{L'émergence des couches moyennes et supérieures salariées}

Avec la transformation de la structure des activités et la salarisation de l'économie, de nouveaux groupes sociaux se constituent. Entre les élites économiques du secteur privé et le reste de la population, les couches moyennes et cadres supérieurs issus du secteur d'État occupent une place réelle dans la nouvelle configuration sociale.

Si ces derniers appartiennent incontestablement aux nouvelles élites sociales (l'importance de leurs statut, social, politique, symbolique et culturel, 
compense aisément la modestie de leurs revenus économiques), les couches moyennes représentent un groupe social nouveau. Dotées de revenus sécurisés et surtout d'un capital scolaire et culturel ( 14 à 20\% de la population active des villes dispose d'un niveau de scolarisation secondaire, 2 à $4 \%$ d'un niveau universitaire), ces nouvelles catégories de population participent à l'introduction de nouveaux modes de vie. À l'encadrement initial assuré par des personnels venus du Nord, se sont substituées de nouvelles élites locales et des cadres intermédiaires natifs du Sahara. Les professions libérales ne sont pas en reste ; les bureaux d'études, cabinets de médecins, officines de pharmaciens se multiplient. Dans la seule wilaya de Biskra, les cadres et professions libérales représentent environ 4000 personnes dont plus de la moitié est domiciliée dans le chef-lieu, représentant environ $4 \%$ de la population active (Annuaire statistique, 1996).

\section{pauvreté \\ 2.7. Structures populaires et nouvelle}

Le dynamisme des élites et des couches moyennes ne doit pas occulter l'existence d'une importante population appartenant aux milieux populaires. La forte salarisation, la taille importante des familles, le faible taux d'activité (30\%), font que souvent une famille de 6 ou 7 personnes vit d'un seul salaire d'ouvrier de l'industrie, d'employé de services ou de l'administration.
L'économie pétrolière joue dans les régions qu'elle touche un rôle important en distribuant une masse monétaire considérable, mais les emplois sont souvent précaires (environ $80 \%$ des emplois de ce secteur) et concernent majoritairement les activités ne nécessitant pas de qualification (techniciens et spécialistes venant du Nord).

Malgré la dynamisation du secteur économique, le taux de chômage est important $(30 \%$ en moyenne), ce qui alimente une situation de pauvreté que n'arrive pas à compenser le développement d'un secteur informel multiforme (revente d'objets divers, emplois non déclarés). Cette situation économique, qui touche toute l'Algérie, fragilise une population, largement majoritaire, dont les salaires sont peu élevés. Le retour aux solidarités familiales, la cohabitation dans un seul logement de plusieurs ménages agnatiques, permettant de partager les revenus salariés et de l'informel, sont des solutions que les milieux populaires mettent en ceuvre pour subvenir, difficilement, à leurs besoins.

Structures sociales verticales (hiérarchies économiques) et horizontales (solidarités traditionnelles), logique d'intérêt économique et de pouvoir local, la réalité urbaine tend à diluer les rapports lignagers et à recomposer le tissu social. Certes les solidarités claniques ont conservé une certaine vigueur mais elles tendent à s'effacer au profit des nouvelles hiérarchies sociales caractérisées par des différences de richesses, de mode de vie, et dans les processus de construction des identités sociales.

\section{3 - LA RECOMPOSITION URBAINE EN COURS}

\subsection{Urbanisation et transformation des tissus urbains}

La morphologie spatiale de la ville saharienne prend souvent la forme d'une agrégation de tissus, correspondant à quatre temps historique d'urbanisation: le ksar ou la cité traditionnelle, la ville coloniale dans les localités où la présence française a été significative, les quartiers spontanés aux constructions plus ou moins finies, et les zones d'urbanisation programmée. Ce modèle évolue à des rythmes différents; le ksar s'efface au profit de l'habitat collectif moderne, les grandes maisons traditionnelles au profit de luxueuses villas reproduisant les modèles du Nord, les anciennes centralités au profit de nouveaux centres (tabl. 2).

\subsection{Les ksour, un déclin inéluctable}

Le $k s a r$ comme forme traditionnelle d'habitat et d'organisation de l'espace est aujourd'hui en pleine

TABL. 2 - LA DISTRIBUTION DES LOGEMENTS DANS LE SAHARA PAR GRANDS TYPES EN 1998

\begin{tabular}{|c|c|c|c|c|c|c|c|c|c|c|c|}
\hline $\begin{array}{l}\text { PARC } \\
\text { LOGEMENT }\end{array}$ & Chef-lieu & \multicolumn{2}{|c|}{$\begin{array}{l}\text { Immeubles } \\
\text { collectifs }\end{array}$} & \multicolumn{2}{|c|}{$\begin{array}{l}\text { Maisons } \\
\text { individuelles }\end{array}$} & \multicolumn{2}{|c|}{$\begin{array}{c}\text { Maisons } \\
\text { traditionnelles }\end{array}$} & \multicolumn{2}{|c|}{ Précaires } & \multicolumn{2}{|c|}{ Non déclarés } \\
\hline Biskra & 30300 & 19,1 & 8,2 & 74,2 & 65,4 & 2,2 & 21,3 & 3,6 & 4,5 & 0,8 & 0,6 \\
\hline Béchar & 23600 & 16,2 & 11,0 & 77,3 & 79,4 & 2,0 & 5,3 & 3,7 & 3,9 & $-0,4$ & $0,5-$ \\
\hline Ouargla & 19000 & 17,3 & 11,9 & 54,3 & 45,0 & 26,0 & 7,7 & 1,1 & 6,8 & 1,5 & 0,8 \\
\hline Ghardaïa & 17500 & 2,4 & 2,7 & 74,4 & 75,3 & 16,2 & 19,4 & 0,4 & 1,4 & 1,7 & 1,3 \\
\hline El Oued & 17400 & 2,3 & 0,7 & 93,7 & 94,3 & 2,3 & 3,0 & 0,6 & 0,6 & 1,2 & 0,7 \\
\hline Tamanrasset & 15835 & 0,6 & 0,4 & 59,1 & 61,9 & 33,9 & 31,7 & 4,9 & 4,9 & 1,4 & 1,2 \\
\hline Adrar & 10350 & 1,2 & 0,2 & 94,9 & 66,0 & 2,3 & 13,1 & 1,2 & 20,4 & $-0,4$ & $0,2-$ \\
\hline Tindouf & 5060 & 13,6 & 12,7 & 81,7 & 82,3 & 1,5 & 1,5 & 3,1 & 3,3 & $-0,2$ & $0,2-$ \\
\hline Touggourt & 4460 & 14,8 & & 75,5 & & 1,0 & & 6,5 & & 2,2 & \\
\hline Illizi & 2750 & 1,0 & & 43,5 & & 40,0 & & 13,2 & & 2,3 & \\
\hline Moyenne Algérie & & \multicolumn{2}{|c|}{16,1} & \multicolumn{2}{|c|}{56,3} & \multicolumn{2}{|c|}{21,1} & \multicolumn{2}{|c|}{5,4} & \multicolumn{2}{|c|}{1,0} \\
\hline
\end{tabular}


mutation, ce qui pose la question de son devenir. Le discours nostalgique dont sont porteurs certains architectes et urbanistes est révélateur de ces transformations et sans doute de la fin d'une forme spatiale. Les processus de ce déclin sont différenciés tant dans leur rythme que dans les formes, signalant à la fois les résistances, la vigueur de certains tissus et la spécificité des réalités locales. Globalement, à l'échelle du Sahara, l'habitat traditionnel représente une proportion de plus en plus marginale reflétant le déclin des ksour. Dans les principales villes du Sud, ce type d'habitat est inférieur à $3 \%$ (Adrar, Béchar, Biskra, Tindouf, El Oued et Touggourt). Seules Ouargla (35\%) Tamanrasset ( $35 \%$ ), Illizi (41\%) et Ghardaïa (17\%), maintiennent un parc relativement important, localisé dans la vieille ville ou dans le cadre de construction plus récente comme à Tamanrasset.

Quatre grands cas de figure illustrent la situation des ksour: le maintien, la déchéance, l'abandon et la destruction. Dans le M'Zab, à El Oued ou encore à Timimoun, la rénovation par les habitants qui reconstruisent parfois sous une forme traditionnelle, mais le plus souvent en ayant recours à des matériaux modernes, permet aux ksour de se maintenir. Le ksar de Ouargla est inégalement entretenu, mais continue d'accueillir une population nombreuse. À El Oued, les parties attenantes au vieux marché font l'objet de transformation qui permet l'extension du marché dans la vieille ville.

Les ksour en déchéance, abandonnés par leurs propriétaires qui s'installent dans les nouveaux quartiers, sont réappropriés par les populations les plus pauvres, les nouveaux immigrants venus des Aurès (El Oued, Ziban) ou les dernières vagues de sédentarisation des nomades (Ouargla). Parfois, ils deviennent de véritables îlots de pauvreté, comme le ksar de Tolga dont l'abandon est déjà partiel.

Les cas sont nombreux où les ksour sont entièrement abandonnés (Farfar et Lichana dans les Ziban, Tamerna ou Temacine dans l'Oued Righ), faisant suite à une lente dégradation, accélérée par les inondations de 1969 et 1990 . Phase finale d'un processus d'abandon ou de déchéance, la destruction dans le cadre d'opération publique d'urbanisme est accompagnée d'un relogement de la population, comme à Touggourt pour le ksar Mestaoua.

La forte densification des ksour est en grande partie responsable de leur dégradation et de la fragilisation de l'habitat. El Oued et Guemar, les deux cités importantes du Souf, ont connu une densification, bien avant l'indépendance. Le $k s a r$ de Timimoun a connu un croît de 2000 personnes de 1952 à 1979. Ces fortes densités ont conduit à la modification de l'habitat et notamment à l'édification de maisons à deux niveaux et l'occupation des cours intérieures (BATAILLON, 1955 ; Bisson, 1983a).

Le ksar Mestaoua de Touggourt, illustre le déclin et le devenir du tissu traditionnel. Déjà amputé d'une partie de son bâti lors de la période coloniale, le ksar a connu une lente dégradation aboutissant à une opération de rénovation qui ne laisse subsister que quelques îlots, en bordure des grandes voies de communication existantes. Au moment de sa destruction, 820 personnes, soit 108 ménages occupaient le ksar ( $P O S$ de Mestaoua, 1997). Sur les 159 habitations recensées, 52 étaient abandonnées, 108 encore occupées, mais 70 d'entre elles présentaient un état de vétusté plus ou moins avancé. De grands axes de communication vont le traverser de part en part pour l'intégrer au tissu de la nouvelle ville. Des programmes d'habitat et d'équipements sont prévus; seul le maintien des vieilles mosquées et les locaux d'une zaouïa rappelleront la grandeur de la capitale des Ben Djellab.

\subsection{Les quartiers spontanés et les extensions} urbaines.

Les quartiers spontanés se développent sous différentes formes : constructions précaires, transformation des lotissements communaux de la période coloniale ou de l'indépendance, construction de nouveaux quartiers sans autorisation. À El Oued, l'arrivée en 1962 de plusieurs milliers de réfugiés, installés en Tunisie pendant la guerre, a été à l'origine d'une première extension de la ville : El Gaouatine, (le quartier des tentes, appelé ainsi en référence aux habitations d'urgence mises en place par les réfugiés). D'autres quartiers, El Asnam et Moustaqbel se sont développés, accueillant les populations des ksour du Souf, d'anciens nomades et des Nememcha des Aurès. À Ouargla, c'est la sédentarisation des nomades chaamba qui aboutit à la formation du quartier Beni Thour, l'un des plus importants de la ville. À Hassi-Messaoud, où l'habitat précaire représente actuellement $7 \%$ de l'ensemble, c'est l'exode d'une population attirée par les emplois des hydrocarbures qui en est le facteur. Progressivement, au bout de plusieurs années, cet habitat précaire se transforme en habitat durable, continuant néanmoins à porter les stigmates de la pauvreté.

\section{publics \\ 3.4. L'habitat collectif et les programmes \\ Les programmes publics de logements} collectifs touchent inégalement les villes du Sud. Encore marginaux à Adrar, Tamanrasset, Illizi, Ghardaïa et El Oued (moins de $2 \%$ du parc logement), ils sont supérieurs ou égaux à la moyenne nationale à Ouargla, Touggourt, Hassi-Messaoud, Tindouf, Biskra et Béchar. Ces programmes de logement qui accompagnent la croissance urbaine et les migrations de population sont destinés à loger prioritairement le personnel d'encadrement des administrations et du secteur public. Ils répondent aussi à la demande locale, notamment en permettant de reloger les 
populations des vieux centres villes sinistrés. Ce nouveau type d'habitat recompose la ville et les formes d'habiter. Les ZHUN (Zone d'habitat et d'urbanisme nouveau) deviennent des centres de vie sociale, avec leurs équipements, leurs commerces et leurs nouveaux espaces de sociabilité.

Dans des villes comme Touggourt, l'habitat collectif connaît une progression continue depuis les années 1960. À Biskra et Béchar, il marque d'une certaine manière le triomphe des modèles d'habitations du Nord, s'inscrivant dans de grands programmes d'urbanisme, véritables villes nouvelles qui recomposent le paysage urbain. À Biskra, les programmes collectifs sont encore loin d'être achevés, 11800 logements collectifs sont en cours de réalisation ou programmés. Dans d'autres villes, il ne fait aucun doute que dans les années à venir le parc collectif va croître, comme à El Oued où 700 logements sont en cours de réalisation et où 3000 demandes sont en instance.

\subsection{Les nouvelles stratégies pavillonnaires}

Comme dans les villes du Nord, 10 ou 20 ans auparavant, le développement urbain entraîne des stratégies résidentielles, encouragées par les politiques publiques, qui participent à la construction des nouvelles identités sociales. La mise en lotissement de nouveaux terrains, avec des plans de charge spécifiques et des modalités de vente particulières, s'inscrit dans les nouveaux projets résidentiels des bourgeoisies et petites bourgeoisies sahariennes. Dans un premier temps de riches maisons étaient construites dans les tissus existants, au sein de quartiers à l'habitat hétérogène. Aujourd'hui, les villes se dotent de quartiers résidentiels où les villas cossues rivalisent de richesse (Er Rimmel à El Oued, la rue de Koweit à Touggourt, les nouveaux lotissements à l'extérieur de l'enceinte de Beni Isguen). Avec ces stratégies pavillonnaires, la spéculation foncière se développe ${ }^{10}$ et les mécanismes de ségrégation économique favorisent l'émergence de quartiers distincts socialement. Riches villas à l'architecture massive et distinctive reflètent les nouvelles réalités urbaines de la ville saharienne et le style de vie qu'adoptent les nouvelles élites sociales.

Mais l'habitat nouveau ne concerne pas seulement les plus riches, il est le fait le plus répandu dans la plupart de ces villes où l'habitat individuel représente entre 54\% (Ouargla) et $94 \%$ (El Oued) du parc logement. Maisons inachevées pour beaucoup, d'un ou deux niveaux, aux façades non traitées, dans des parcellaires plus ou moins prêtes à être surélevées dès que les revenus le permettront; maisons familiales à trois étages, souvent dotées de garages déjà loués pour abriter des fonctions marchandes ou d'artisanat, elles sont l'élément dominant du nouveau paysage urbain.

\subsection{Les plans d'urbanisme ou les nouveaux desseins urbains}

Les politiques publiques d'urbanisme sont mues par une logique d'ensemble recourant aux mêmes outils de gestion urbaine que dans le Nord (PDAU, Plan d'architecture et d'urbanisme et POS, Plan d'occupation des sols ). Elles consistent en une programmation des sols à urbaniser, une intégration des espaces déjà urbanisés, une distribution des équipements et de l'habitat. À partir de grands axes de communication, qui ont un rôle structurant, les tissus déjà existants sont enserrés, et peuvent faire l'objet d'intervention, notamment par la mise en place des réseaux de viabilisation. En enserrant la ville existante et les quartiers spontanés, ils évitent leur extension et permettent de les canaliser. Ils organisent la distribution du foncier dans le cadre de programmes d'habitat qui se répartissent entre collectif et lotissements individuels. Les PDAU, pcrmettent un quadrillage spatial, relativement efficace et dessinent les nouvelles linéarités et centralités urbaines. La gestion «technicienne» de la ville laisse peu de place à des procédures de concertation avec la population, et l'élaboration des projets reste confinée à l'intérieur des appareils de gestion et de décision. Pourtant à El Oued et dans le M'Zab, d'intéressantes formes de «gouvernances urbaines», préfigurent l'émergence d'une société civile participant timidement aux projets de ville. De nouveaux centres villes apparaissent, confinant les vieux $k$ sour à des fonctions symboliques (M'Zab), commerciales (El Oued) ou les laissant à l'abandon (Touggourt). La ville fonctionnelle l'emporte sur la ville traditionnelle. La fragmentation de la centralité voulue par les plans d'urbanisme distribue sur des espaces de plus en plus grand, souvent à partir d'une logique linéaire et de zonage, les différentes fonctions de la ville.

\subsection{Du passage de la médina à la ville} moderne: la confrontation entre deux logiques urbaines

Le passage de l'habitat en médina à l'habitat en ville «moderne» interroge sur les conséquences induites sur les modes de vie, de sociabilité, de pratique de la ville; en un mot sur l'urbanité nouvelle que cela engendre.

La détérioration du tissu urbain et de l'habitat des ksour, le formidable développement de ces localités, lié à la forte croissance démographique et au desserrement de l'habitat, lui-même généré par la transformation des structures familiales, l'expansion spatiale de ces localités qui depuis un certain temps sont sorties de leurs murs, les nouveaux modes de vie qui entraînent des exigences nouvelles par rapport à l'habiter, au confort, à l'utilisation des espaces de la maison, obligent à réfléchir sur leur devenir et sur le projet urbain. 
L'urbanisme, en tant que modèle d'organisation des villes n'est jamais neutre, et derrière chaque dessin urbain se cache un dessein social et politique. Entre la ligne droite et la courbe, entre l'orthogonalité et les cercles concentriques, entre l'îlot et la barre, des réponses sont à trouver. En Algérie, la question urbaine et les réponses qui leur sont apportées restent empreintes d'un modèle d'urbanisation et de logement qui est celui mis en place en Europe avec l'avènement du capitalisme industriel fordiste. Les ZHUN qui croissent dans les villes sahariennes, où cohabitent habitat collectif, lotissements pavillonnaires et habitat spontané, reconfigurent l'urbain. Mais s'agit-il d'un modèle universel ?

Certes la détérioration du tissu urbain et de l'habitat traditionnel, les exigences économiques et la non-fonctionnalité des ksour obligent à des interventions et posent la question de quel(s) type(s) de rénovation. En Europe, au nom des conceptions «hygiénistes» développées à la limite $\mathrm{XIX}^{\mathrm{e}} / \mathrm{XX}^{\mathrm{e}}$ siècles, des pans entiers du patrimoine urbain et architectural des villes ont disparu. Les rénovations mises en œuvre ont abouti à des restructurations dont la matrice est la ségrégation sociospatiale. Région en pleine mutation, marquée par un phénomène urbain nouveau et très fort, le Sahara vit une transition sociétale. La ville usage, celle de l'œuvre et du signe, est devenue la ville échange, celle du règne des promoteurs, de la fonctionnalité et de la segmentation des espaces, des temps de vie et des hiérarchies sociales. L'urbanisme saharien a longtemps été un urbanisme de signes, comment s'adaptera-t-il aux nouvelles logiques?

\section{BIBLIOGRAPHIE}

Annuaire statistique de la wilaya de Biskra, (1996), 97 p.

Batalllon Cl., (1955), Le Souf, étude de géographie humaine, Ed. Imbert, Alger, $140 \mathrm{p}$.

Ben Mansour A. et H., (1998), Alger $x v I^{e}-x v i I^{\circ}$ siecle, Journal de Jean-Baptiste GramaYe, évêque d'Afrique, Ed. du CERF, Paris, 769 p.

Berque J., (1958), La cité éminente, p.49-63, Cité et volonté, le donné et le construit, p.170-178, in Les villes, entretiens interdisciplinaires sur les sociétés musulmanes, Ecole Pratique des Hautes Études, Paris.

Bısson J., (1983a), L'industrie, la ville, la palmeraie au désert. Un quart de siècle d'évolution au Sahara algérien, $M a$ ghreb Machrek, $\mathrm{n}^{\circ}$ 99, p.5-79.

Bisson J., (1983b), Les villes sahariennes, politique volontariste et particularismes régionaux, Maghreb Machrek, $n^{\circ} 100$, p.25-41.

Bourdieu P., (1980), Questions de sociologie, Ed. de Minuit, Paris, $268 \mathrm{p}$.

Bureau d'Etudes Architecture et Techniques, (1997), pOS rénovation de Mestaoua, Touggourt, $101 \mathrm{p}$.

Depont O., Coppolani X., (1897), Les confréries religieuses musulmanes, Adolphe Jourdan, Alger, $576 \mathrm{p}$.

El BeKrI, (1913), L'Afrique septentrionale, Adolphe Jourdan, Alger, $405 \mathrm{p}$.

Ibn Hauqal, (1964), Configuration de la terre, Kitab surat alard, Ed. Maisonneuve et Larose, 2 vol., 550 p.

Ibn Khaldoun, (1982), Histoire des Berbères et des dynasties musulmanes de l'Afrique septentrionale, nouvelle éd. Geuthner, Paris, 4 vol., 444 p., 594 p., 494 p., 630 p.

Jean - Léon L'Africain, (1980), Description de l'Afrique, AdrienMaisonneuve, Paris, 2 vol., 630 p..

LethielleuX J., (1983), Ouargla, cité saharienne, des origines au début $d u x X^{e}$ siècle, Geuthner, Paris, 298 p.

Najah A., (1971), le Souf des oasis, Ed. la Maison des Livres, Alger, 174 p.

Office National des Statistiques, (1998), Armature urbaine, RGPH 1998, Collections statistiques, $\mathrm{n}^{\circ} 97$, Alger, $95 \mathrm{p}$.

Shaw T., (1980), Voyage dans la Régence d'Alger, Ed. Bouslama, Tunis, 402 p.

Venture de Paradis, (1983), Tunis et Alger au xvm' e siècle, la bibliothèque arabe, Sindbad, Paris, $287 \mathrm{p}$.

\section{Notes}

1- Problématique actuellement développée par l'équipe de recherche «Urbanisation au Sahara». M. Cóte en est l'inspirateur. 2-L'urbanité est une notion sociologique qui renvoie aux modes d'appropriation des espaces urbains, aux pratiques sociales, aux statuts sociaux de ses habitants, aux modes de vie induits par la ville. Ainsi chaque type d'urbanisation génère des formes d'urbanité, des façons de vivre la ville, de la pratiquer, de se l'approprier, de se la représenter. L'urbanité, c'est à la fois le rapport à l'habiter, à la vie publique, à la sociabilité, aux activités économiques et sociales.

3- Pour Tamanrasset le taux de croissance urbaine est exagéré. Le recensement de 1987 avait compté à part l'agglomération de Tahagart qui appartenait déjà au même tissu urbain.

4- Seul LÉon L'Africain au XVI siècle avancera une estimation chiffrée de 2500 maisons pour Touggourt.

5- Ainsi au XVI ${ }^{e}$ siècle, le Dey Salah Raïs prend Touggourt qui est livrée au pillage. 12000 personnes sont vendues en esclavage Il impose à la cité un tribu annuel qui comportait la livraison de 15 esclaves noirs, la ville de Ouargla devra en livrer 30 (LethielleuX, 1983).
6- Le rituel du marché quotidien de Beni Isguen, la cité sainte du M'Zab, entretient aujourd'hui encore la symbolique du contrôle religieux dans l'échange économique.

7- À titre d'exemple, Depont et Coppolani (1897) dénombrent à la fin du $X_{1} x^{e}$ siècle 5 confréries dans le seul cercle de Biskra. Parmi elles, la Rahmania, de loin la plus importante compte près de 5000 adeptes, 92 Tolba, 7 Moqqadem et 16 Chouikh. À El Oued la Rahmania compte 7 zaouia et 2500 adeptes.

8- Les Juifs qui restèrent à Ghardaïa comme à Touggourt jusqu'en 1962, continueront pendant près de 5 siècles à considérer Tamentit (qui fut un important marché du Touat) comme leur Jérusalem africaine. Chaque année, au jour de la Pâque, ils se saluaient avec cette formule «Plaise à Dieu, l'an prochain à Tamentit» (LeThielleuX, 1983).

9- Les groupes de rencontre qui s'improvisent à tout moment, et où l'on s'assoit à même le sol, sur les trottoirs et les places des différents quartiers des villes, les cafés fréquentés, la possession de chèvres à l'intérieur des maisons sont des éléments marquant cette citadinité paysanne.

10- À El Oued, les lots à bâtir de la route de Touggourt sont vendus dix fois plus cher que ceux du quartier El Chott. 